\title{
Effect of plasticizer content on the functional properties of extruded gelatin-based composite films
}

\begin{abstract}
Beef gelatin, in combination with varying levels of glycerol, was used to manufacture films by extrusion. A twin-screw co-rotating extruder was employed to produce the films and the mechanical and barrier properties of the films were investigated. Increasing the plasticizer content increased $(\mathrm{P}<0.05)$ elongation at break $(\mathrm{EAB})$ values but decreased $(\mathrm{P}<0.05)$ tensile strength (TS) values. Oxygen permeability (OP) values for gelatin-based composite films increased $(\mathrm{P}<0.05)$ as the concentration of glycerol increased. Additionally, the solubility of films in water and seal strength increased as glycerol content increased. FTIR results indicated that increasing glycerol concentration increased and displaced the peak situated around $1032 \mathrm{~cm}-1$, which corresponded to glycerol. Gelatin-based composite films with a concentration of $0.2 \%$ glycerol possessed the lowest water vapor permeability (WVP) and OP values. From the data generated in this study, it is clear that the use of a plasticizing agent in film formulations should be carefully considered because of the negative effects that the plasticizing agent could have on extruded film barrier properties.
\end{abstract}

Keyword: Gelatin; Composite films; Extrusion; Plasticizer; FTIR 